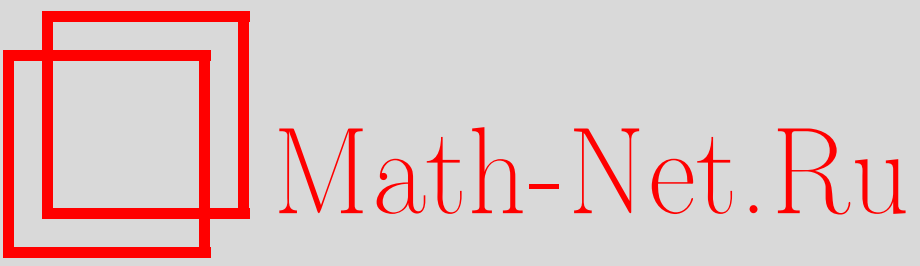

Ю. П. Вирченко, Л. П. Данилова, Графы и алгебры симметрических функций, Итоги науки и техн. Сер. Соврем. мат. и ее прил. Темат. обз., 2020, том $174,20-36$

DOI: https://doi.org/10.36535/0233-6723-2020-174-20-36

Использование Общероссийского математического портала Math-Net.Ru подразумевает, что вы прочитали и согласны с пользовательским соглашением

http://www. mathnet.ru/rus/agreement

Параметры загрузки:

IP: 176.194 .166 .118

27 мая 2020 г., 14:01:15 


\title{
ГРАФЫ И АЛГЕБРЫ СИММЕТРИЧЕСКИХ ФУНКЦИЙ
}

\author{
(c) 2020 г. . П. ВИРЧЕНКО, Л. П. ДАНИЛОВА
}

\begin{abstract}
АннотАция. Излагается алгебраическая техника оперирования со степенными рядами, коэффициенты которых представляются интегралами от симметрических функций $f_{n}$, определенных на декартовых степенях $\Omega^{n}$ множества $\Omega$ с мерой $\mu$. При этом каждая из коэффициентных функций $f_{n}$ получается посредством специального отображения из графов с $n$ помеченными вершинами, принадлежащих фиксированному классу. Эта техника имеет применение в равновесной статистической механике и к задачам перечисления графов.
\end{abstract}

Ключевые слова: граф, коммутативная алгебра, симметрическая функция, инвариантная мера, порождающая функция, мультипликативный функционал.

\section{GRAPHS AND ALGEBRAS OF SYMMETRIC FUNCTIONS}

\author{
(C) 2020 YU. P. VIRCHENKO, L. P. DANILOVA
}

\begin{abstract}
We describe an algebraic technique for operating with power series whose coefficients are represented by integrals of symmetric functions $f_{n}$ defined on the Cartesian powers $\Omega^{n}$ of a set $\Omega$ with a measure $\mu$. Moreover, each of the coefficient functions $f_{n}$ is obtained by means of a special mapping from graphs with $n$ labeled vertices belonging to a fixed class. This technique has application to equilibrium statistical mechanics and to problems of enumeration of graphs.
\end{abstract}

Keywords and phrases: graph, commutative algebra, symmetric function, invariant measure, generating function, multiplicative functional.

AMS Subject Classification: 82B05, 05C30, 13A99

1. Введение. В 1938 г. Дж. Майер предложил описывать структуру коэффициентов степенных рядов, возникающих в задачах статистической механики газов, посредством графов (см. $[11,12])$. Использованная в этих работах конструкция довольно прозрачно реализуется в случае так называемых групповых разложений по степеням активности (см. [4]). Для формул, определяющих их коэффициенты, имеются достаточно полные математические доказательства (см., например, $[5,8])$. Однако используемая при этом алгебраическая техника допускает обобщение и поэтому может быть приложена к гораздо более широкому кругу задач математической физики. В частности, она пригодна для для вычисления коэффициентов так называемых вириальных разложений статистической механики, которые остаются малоизвестными (см. [3]) и для которых не имеется строгого доказательства в математической литературе. Восполнению этого пробела посвящена настоящая работа. План изложение следующий. В разделе2 приводятся необходимые сведения из теории графов с помеченными вершинами: вводятся понятия и формулируются их свойства. Доказательства при этом опускаются, либо указывается путь, на котором они могут быть получены. За разъяснениями мы отсылаем читателя к известным монографиям по теории графов (см., например, $[6,9])$. В разделе 3 кратко излагаются сведения о бесконечномерных коммутативных алгебрах последовательностей симметрических функций. В разделе 4 
устанавливается связь между графами с помеченными вершинами и симметрическими функциями. Последний раздел посвящен доказательству формулы, которая играет основную роль при построении вириальных разложений.

2. Графы с помеченными вершинами. Пусть $V$ - конечное множество элементов, которые называются вершинами; далее обозначаем их малыми латинскими буквами. Обозначим через $V^{(2)}$ множество всех пар $\{x, y\} \subset V$. Графом с помеченными вериинами (далее просто графом) над множеством $V$ называется упорядоченная пара $\mathfrak{G}=\langle V, \Psi\rangle$, где подмножество $\Psi \subset V^{(2)}$ называется множеством смежности графа, а его элементы - ребрами графа $\langle V, \Psi\rangle$. В теории графов, рассматривают также графы, у которых вершины не обладают метками; они определяются как фактор-множество $\langle V, \Psi\rangle / \mathbb{P}_{|V|}$ по группе перестановок $\mathbb{P}_{|V|}$. Граф $\mathfrak{G}^{\prime}=\left\langle V^{\prime}, \Psi^{\prime}\right\rangle$, у которого $V^{\prime} \subset V$, $\Psi^{\prime}=\Psi \cap V^{\prime(2)}$ называется подграфом графа $\mathfrak{G}$.

Бинарное симметричное отношение $\Psi$ смежности на $V$ определяет бинарное отношение связности на $V$. Оно конструируется на основе понятия $n y m u$ на графе $\mathfrak{G}=\langle V, \Psi\rangle$. Последовательность $\gamma(x, y)=\left\langle x, x_{1}, x_{2}, \ldots, x_{n-1}, y\right\rangle$ вершин из $V$, для которой $\left\{x_{j}, x_{j+1}\right\} \in \Psi, j=0,1, \ldots, n-1$, $x_{0}=x, x_{n}=y$ называется путем с множеством вершин $\{\gamma(x, y)\}$. Пара $\{x, y\} \subset V$ называется связной на графе $\mathfrak{G}$, если существует путь $\left\langle x, x_{1}, x_{2}, \ldots, x_{n-1}, y\right\rangle$, содержащий эти вершины. Подмножество пар связных вершин генерирует бинарное отношение, которое обладает свойствами симметричности и транзитивности и, таким образом, является отношением эквивалентности. Тогда оно генерирует разбиение графа на связные попарно непересекающиеся связные компоненты графа.

Пусть $\mathfrak{G}_{1}=\left\langle V_{1}, \Psi_{1}\right\rangle$ и $\mathfrak{G}_{2}=\left\langle V_{2}, \Psi_{2}\right\rangle$ - связные подграфы связного графа $\mathfrak{G}=\langle V, \Psi\rangle$, причем

$$
V_{1} \cap V_{2}=\{x\}, \quad V=V_{1} \cup V_{2} ; \quad \Psi=\Psi_{1} \cap \Psi_{2}=\varnothing, \quad \Psi=\Psi_{1} \cup \Psi_{2} .
$$

Тогда $x$ называется вершиной сочленения графа $\mathfrak{G}$. Граф, не имеющий вершин сочленения, будем в дальнейшем называть блоком. По определению, любой одновершинный граф $\{x\}$ не имеет вершины сочленения. Если $x$ - вершина сочленения графа $\mathfrak{G}$, так что имеют место равенства $(2.1)$,

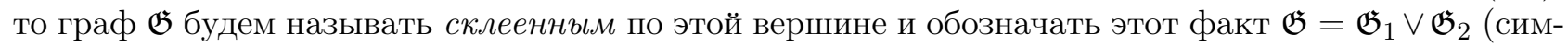
вол $\vee$ обозначает операцию склейки). Вершина сочленения в связном графе $\mathfrak{G}$ характеризуется следующим свойством.

Теорема 2.1. Для того чтобъ вериина $x$ связного графа $\mathfrak{G}=\langle V, \Psi\rangle$ была верииной сочленения, необходимо и достаточно чтобъ существовала пара вершин $y_{1} \in V, y_{2} \in V, y_{j} \neq x$, $j=1,2$, для которой любой путь $\gamma\left(y_{1}, y_{2}\right)$ из $y_{1}$ в $y_{2}$ обязательно содержит вершину $x$.

Если вершина $x$ является вершиной сочленения в графе $\mathfrak{G}$ и этот граф представляется в виде склейки

$$
\mathfrak{G}=\bigvee_{j=1}^{p} \mathfrak{G}_{j}
$$

таких связных графов $\mathfrak{G}_{j}=\left\langle V_{j}, \Psi_{j}\right\rangle, j=1, \ldots, p$, что

$$
V_{j} \cap V_{k}=\{x\}, \quad \Psi_{j} \cap \Psi_{k}=\varnothing, \quad j \neq k ; \quad j, k=1, \ldots, p
$$

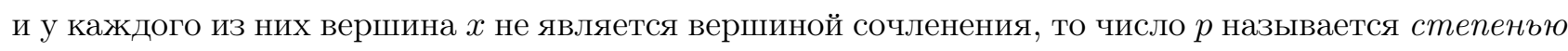
вершины сочленения $x$ на графе $\mathfrak{G}$. При этом графы $\mathfrak{G}_{j}$ будем называть компонентами, соответствующими вершине сочленения $x$. Если вершина $x$ не является вершиной сочленения то, по определению, будем считать, что ее степень равна 1. Каждой вершине сочленения соответствует определенная степень.

Теорема 2.2. Пусть $x$ - вершина сочленения связного графа $\mathfrak{G}=\langle V, \Psi\rangle$. Тогда существуют такие число $s \geqslant 2$ и однозначно определенный набор связных графов $\mathfrak{G}_{j}=\left\langle V_{j}, \Psi_{j}\right\rangle, j=1, \ldots, s$, в каждом из которых вершина $x$ уже не является вершиной сочленения, для которых имеют место следующие соотношения:

$$
\begin{aligned}
& V=V_{1} \cup V_{2} \cup \cdots \cup V_{s}, \quad V_{i} \cap V_{j}=\{x\}, \quad i \neq j, \quad i, j=1, \ldots, s ; \\
& \Psi=\Psi_{1} \cup \Psi_{2} \cup \cdots \cup \Psi_{s}, \quad \Psi_{i} \cap \Psi_{j}=\varnothing, \quad i \neq j, \quad i, j=1, \ldots, s .
\end{aligned}
$$


Следствие 2.1. В любом конечном неодновершинном графе имеется, по крайней мере, две вершины, которые не являются его вериинами сочленения.

Введем более общее понятие склейки двух графов. Пусть два графа $\mathfrak{G}_{j}=\left\langle V_{j}, \Psi_{j}\right\rangle, j=1,2$, таковы, что $V_{1} \cap V_{2} \neq \varnothing$. Склейкой $\mathfrak{G}_{1} \vee \mathfrak{G}_{2}$ этих графов назовем граф $\left\langle V_{1} \cup V_{2}, \Psi_{1} \cup \Psi_{2}\right\rangle$. Это понятие дает возможность ввести следующую конструкцию.

Пусть в графе $\mathfrak{G}=\langle V, \Psi\rangle$ имеется подграф $\mathfrak{G}_{B}=\left\langle B, \Psi_{B}\right\rangle, \Psi_{B}=\Psi \cap B^{(2)}$, который не имеет вершин сочленения и для которого существует набор $\{\mathfrak{G}(z)=\langle V(z), \Psi(z)\rangle ; z \in B\}$ связных, но попарно несвязных друг с другом подграфов графа $\mathfrak{G}$, обладающих следующими свойствами: $B \cap V(z)=\{z\}, \Psi_{B} \cap \Psi(z)=\varnothing, z \in B$, и граф $\mathfrak{G}$ представим в виде

$$
\mathfrak{G}=\bigvee_{z \in B}\left[\mathfrak{G}_{B} \vee \mathfrak{G}(z)\right]
$$

где допускается, что некоторые из подграфов $\mathfrak{G}(z), z \in B$, могут быть пустыми. Тогда будем говорить, что подграф $\mathfrak{G}_{B}$ является блоком в составе графа $\mathfrak{G}$.

Следующее утверждение, ввиду существования в каждом графе вершины, не являющейся вершиной сочленения, гарантирует существование содержащегося в нем блока.

Теорема 2.3. Пусть $x$ - вершина графа $\mathfrak{G}=\langle V, \Psi\rangle$, не являющаяся вершиной сочленения. Тогда в графе $\mathfrak{G}$ найдется единственный блок $\mathfrak{G}_{B}=\left\langle B, \Psi_{B}\right\rangle$, для которого справедливо представление (2.2) и который содержит $x$.

Следующее утверждение уточняет формулу (2.2).

Теорема 2.4. Пусть $x$-вершина связного графа $\mathfrak{G}=\langle V, \Psi\rangle$, которая не является его вершиной сочленения, $u \mathfrak{G}_{B}=\left\langle B, \Psi_{B}\right\rangle$-единственный блок в этом графе, который содержит эту вериину. Тогда граф $\mathfrak{G}$ представим в виде

$$
\mathfrak{G}=\bigvee_{z \in B}\left[\mathfrak{G}_{B} \vee\left(\bigvee_{j=1}^{p(z)-1} \mathfrak{G}_{j}(z)\right)\right],
$$

где $\mathfrak{G}_{B}-$ блок в графе $\mathfrak{G}$, которому принадлежит $x$, и для каждой вершины $z \in B$ числа $p(z)$ являются их степенями в графе $\mathfrak{G}$, а связные графы $\mathfrak{G}_{j}(z), j=1, \ldots, p(z),-$ компонентами сочленения, соответствующими вершине z.

Разложсением $\mathcal{A}$ множества $I_{n}=\{1, \ldots, n\}$ называется дизъюнктивный набор $\left\{\Gamma_{1}, \ldots, \Gamma_{s}\right\}$ подмножеств из $I_{n}$, называемых компонентами, $\Gamma_{j} \cap \Gamma_{k}=\varnothing$ при $j \neq k$, для которого $\bigcup_{j=1}^{s} \Gamma_{j}=I_{n}$, число $s \equiv|\mathcal{A}|$ называется порядком разложения. Класс всех разложений порядка $s$ множества $I_{n}$ будем обозначать $\mathfrak{S}_{n}^{(s)}$, а класс всех разложений $-\mathfrak{S}=\bigcup_{s=1}^{n} \mathfrak{S}_{n}^{(s)}$. Здесь классы $\mathfrak{S}_{n}^{(1)}$ и $\mathfrak{S}_{n}^{(n)}$ состоят из одного разложения, соответственно, $\mathfrak{S}_{n}^{(1)}=\left\{I_{n}\right\}$ и $\mathfrak{S}_{n}^{(n)}=\left\{\Gamma_{j}=\{j\}, j=1, \ldots, n\right\}$.

Пусть $\overline{\mathcal{G}}[V ; z]$ - класс всех связных графов над множеством вершин $V \cup\{z\}$ с отмеченной вершиной $z$. Этот класс является подклассом среди класса всех связных графов над $V \cup\{z\}$. Он характеризуется свойством инвариантности относительно перенумераций вершин из $V$. А именно, пусть $\mathrm{P}$ принадлежит группе $\mathbb{P}_{|V|}$ перестановок множества $V$. Тогда номера вершины $z$ в множествах $\mathrm{P} V \cup\{z\}$ и $V \cup\{z\}$ совпадают. Перенумерация $\mathrm{P}$ индуцирует преобразование $\mathrm{P} \Psi \equiv\{\{\mathrm{P} x, \mathrm{P} y\}:\{x, y\} \in \Psi\}$ множества смежности $\Psi$ у каждого графа $\mathfrak{G}=\langle V \cup\{z\}, \Psi\rangle$ и, следовательно, - преобразование $\mathrm{P} \mathfrak{G}=\langle\mathrm{P} V \cup\{z\}, \mathrm{P} \Psi\rangle$ любого графа $\mathfrak{G} \in \overline{\mathcal{G}}[V \cup\{z\}]$. Тогда свойство инвариантности класса $\overline{\mathcal{G}}[V ; z]$ относительно Р означает, что

$$
\mathrm{P} \overline{\mathcal{G}}[V ; z] \equiv\{\mathrm{P} \mathfrak{G} ; \mathfrak{G} \in \overline{\mathcal{G}}[V ; z]\}=\overline{\mathcal{G}}[V ; z] .
$$

Ниже нам понадобятся следующие технические леммы, доказательства которых очевидны. 
Лемма 2.1. Класс $\overline{\mathcal{G}}\left[I_{n} ; n+1\right], n \in \mathbb{N}$, представим в виде дизгюнктивного объединения

$$
\overline{\mathcal{G}}\left[I_{n} ; n+1\right]=\bigcup_{s=1}^{n} \overline{\mathcal{G}}^{(s)}\left[I_{n} ; n+1\right],
$$

где $\overline{\mathcal{G}}^{(s)}\left[I_{n} ; n+1\right]$ - класс всех связных грабов с множеством вершин $I_{n+1}$, у которых отмеченнал вериина является верииной сочленения степени $s=1, \ldots, n$.

При этом очевидно, что $\mathrm{P} \overline{\mathcal{G}}^{(s)}\left[I_{n} ; n+1\right]=\overline{\mathcal{G}}^{(s)}\left[I_{n} ; n+1\right], \mathrm{P} \in \mathbb{P}_{n}$.

Лемма 2.2. Если отмеченная вершина $n+1$ является вершиной сочленения со степенъю $s>1$, то класс $\overline{\mathcal{G}}^{(s)}\left[I_{n} ; n+1\right]$ представим в виде дизгюнктивного обгединения

$$
\overline{\mathcal{G}}^{(s)}\left[I_{n} ; n+1\right]=\bigcup_{\mathcal{A} \in \mathfrak{S}_{n}^{(s)}} \overline{\mathcal{G}}^{(s)}\left[I_{n} ; \mathcal{A}, n+1\right]
$$

с непустыми компонентами, каждая из которых представляет собой класс всех связанных графов над множеством $I_{n+1}$ с отмеченной вершиной $n+1$ со степенью сочленения $s$. При этом номера вершин, отличные от $n+1$, у свлзных графов $\mathfrak{G}_{j}, j=1, \ldots, s,-$ компонент сочленения в вершине $n+1$ образуют разложение $\mathcal{A} \in \mathfrak{S}_{n}^{(s)}$ с числом компонент $s$, а вершина $n+1$ не является верииной сочленения в графах $\mathfrak{G}_{j}$.

Каждый из классов $\overline{\mathcal{G}}^{(s)}\left[I_{n} ; \mathcal{A}, n+1\right], \mathcal{A} \in \mathfrak{S}_{n}^{(s)}$, инвариантен относительно таких перенумераций $\mathrm{P} \in \mathbb{P}_{n}$ множества $I_{n}$, которые не изменяют разложения $\mathcal{A}$, т.е.

$$
\mathrm{P} \mathcal{A}=\left\langle\mathrm{P} A_{j} ; j=1, \ldots, s\right\rangle=\left\langle A_{j} ; j=1, \ldots, s\right\rangle \equiv \mathcal{A} .
$$

Лемма 2.3. Для любого дизгюнктивного разложения $\mathcal{A}=\left\{A_{1}, \ldots, A_{s}\right\} \in \mathfrak{S}_{n}^{(s)}$ класс графов $\overline{\mathcal{G}}^{(s)}\left[I_{n} ; \mathcal{A}, n+1\right], n \geqslant s>1$, эквивалентен декартову произведению

$$
\overline{\mathcal{G}}^{(s)}\left[I_{n} ; \mathcal{A}, n+1\right]=\bigotimes_{A \in \mathcal{A}} \overline{\mathcal{G}}^{(1)}[A ; n+1]
$$

где $\overline{\mathcal{G}}^{(1)}[A ; n+1]-$ класс связанных графов с множеством вершин $A \cup\{n+1\}, A \in \mathcal{A}$, у которых вершина $n+1$ не является вершиной сочленения.

Каждый из классов $\overline{\mathcal{G}}^{(1)}[A ; n+1]$ инвариантен относительно перенумераций $\mathrm{P} \in \mathbb{P}_{n}$ вершин, которые переводят множество $A \in \mathcal{A}$ в себя, $\mathrm{P} A=A, A \in \mathcal{A}$.

На основе формулы (2.3) доказывается следующее утверждение.

Лемма 2.4. Класс $\overline{\mathcal{G}}^{(1)}\left[I_{n} ; n+1\right], n \geqslant 2$, представим в виде дизгюнктивного обгединения

$$
\overline{\mathcal{G}}^{(1)}\left[I_{n} ; n+1\right]=\bigcup_{B \subset I_{n}:|B| \geqslant 1} \bigcup_{C \subset B} \overline{\mathcal{G}}\left[I_{n} ; B ; C\right]
$$

непустых классов $\overline{\mathcal{G}}\left[I_{n} ; B ; C\right]$ графов с отмеченной вершиной $n+1$, которая не является верииной сочленения. У каждого графа $\mathfrak{G} \in \overline{\mathcal{G}}\left[I_{n} ; B ; C\right]$ непустое множество $B$ состоит из номеров вершин того блока $\mathfrak{G}_{B}$, который содержит вершину $n+1$, а $C$-множество вершин сочленения графа со степенью сочленения, большей 1 , которые содержатся в блоке $\mathfrak{G}_{B}$.

Каждый из классов $\overline{\mathcal{G}}\left[I_{n} ; B ; C\right]$ инвариантен относительно перенумераций $\mathrm{P}$ вершин, которые переводят множества $B$ и $C$ в себя.

Для каждой пары множеств $B \subset I_{n}$ и $C \subset B$ обозначим через $\mathfrak{D}(B, C)$ класс функций $\{B(z) ; z \in C\}$ на $C$, где совокупность значений составляет дизъюнктивное разложение $\bigcup_{z \in C} B(z)=I_{n} \backslash B, B(z) \neq \varnothing, z \in C$ и $B\left(z_{1}\right) \cap B\left(z_{2}\right)=\varnothing$ при $z_{1} \neq z_{2}$. Справедливо следующее утверждение. 
Лемма 2.5. Каждый класс $\overline{\mathcal{G}}\left[I_{n} ; B ; C\right], n \geqslant 2$, представим в виде дизгюнктивного оббединения

$$
\overline{\mathcal{G}}\left[I_{n} ; B ; C\right]=\bigcup_{\{B(z) ; z \in C\} \in \mathfrak{D}(B, C)} \overline{\mathcal{G}}\left[I_{n} ; B \mid\{B(z), z \in C\}\right]
$$

maких непустых классов $\overline{\mathcal{G}}\left[I_{n} ; B \mid\{B(z), z \in C\}\right],\{B(z) ; z \in C\} \in \mathfrak{D}(B, C)$, что каждый граф $\mathfrak{G} \in \overline{\mathcal{G}}\left[I_{n} ; B \mid\{B(z), z \in C\}\right]$ с множествами $B$ и $C$, определенными в лемме 2.4, для которых y каждой вершины $z \in C$ граф, приклеенный $\kappa$ блоку $\mathfrak{G}_{B}$, имеет множество вершин $B(z)$.

Здесь каждый из классов $\overline{\mathcal{G}}\left[I_{n} ; B \mid\{B(z), z \in C\}\right]$ инвариантен относительно перенумераций $\mathrm{P}$ вершин, которые переводят множества $B$ и $C$ в себя и при этом не изменяют элементов разложения $\{B(z), z \in C\}, \mathrm{P} B(z)=B(z), z \in C$.

Лемма 2.6. Каждый класс $\overline{\mathcal{G}}\left[I_{n} ; B \mid\{B(z), z \in C\}\right], n \geqslant 2$, представим в виде декартова произведения

$$
\overline{\mathcal{G}}\left[I_{n} ; B \mid\{B(z), z \in C\}\right]=\mathcal{F}[B ; n+1] \otimes\left(\bigotimes_{z \in C} \overline{\mathcal{G}}[B(z) ; z]\right)
$$

класса $\mathcal{F}[B ; n+1]$ графов без вершин сочленения над множеством вершин $B \cup\{n+1\}$ и набора непустых классов $\overline{\mathcal{G}}[B(z) ; z], z \in C$, где каждый класс состоит из всех связных графов над множеством вершин $B(z) \cup\{z\}$ с выделенной вершиной $z$.

Здесь каждый из классов $\overline{\mathcal{G}}[B(z) ; z]$ для фиксированной вершины $z \in C$ инвариантен относительно перенумераций $\mathrm{P}$ вершин, которые переводят множество $B(z)$ в себя.

3. Алгебры симметрических функций. Пусть фиксировано множество $\Omega$, элементы которого будем обозначать буквами $x, y, z, \ldots$; упорядоченный набор $\left\langle x_{1}, x_{2}, \ldots, x_{n}\right\rangle \in \Omega^{n}$ обозначим $X_{n}$. Функция $f_{n}\left(X_{n}\right), n \geqslant 2$, на $\Omega^{n}$ со значениями в $\mathbb{C}$ называется симметрической, если для любой перестановки $\mathrm{P}$ из группы $\mathbb{P}_{n}$ перестановок множества $I_{n}, n \in \mathbb{N}$, имеет место формула $f_{n}\left(\mathrm{P} X_{n}\right)=f_{n}\left(X_{n}\right)$. Множество всех симметрических функций на $\Omega^{n}$ образует линейное многообразие $\mathbb{L}_{n}(\Omega)$. Рассмотрим прямую сумму

$$
\mathbb{L}_{\infty}(\Omega)=\bigoplus_{n=0}^{\infty} \mathbb{L}_{n}(\Omega)
$$

линейных многообразий: $\mathbb{L}_{0}(\Omega) \equiv \mathbb{C}, \mathbb{L}_{1}(\Omega)$ - линейное многообразие функций $f_{1}\left(x_{1}\right)$ на $\Omega$ и $\mathbb{L}_{n}(\Omega)$ - линейные многообразия симметрических функций $f_{n}\left(X_{n}\right)$ на $\Omega^{n}, n \geqslant 2$. Таким образом, $\mathbb{L}_{\infty}(\Omega)$ состоит из последовательностей $\mathrm{f}=\left\langle f_{n}\left(X_{n}\right) ; n \in \mathbb{N}_{+}\right\rangle$.

На линейном многообразии $\mathbb{L}_{\infty}(\Omega)$ введем отображение $\mathbb{L}_{\infty} \times \mathbb{L}_{\infty} \mapsto \mathbb{L}_{\infty}$, которое каждой паре последовательностей $\mathrm{f}^{(1)}=\left\langle f_{n}^{(1)} ; n \in \mathbb{N}_{+}\right\rangle$и $\mathrm{f}^{(2)}=\left\langle f_{n}^{(2)} ; n \in \mathbb{N}_{+}\right\rangle$сопоставляет однозначным образом последовательность $\mathrm{f}=\left\langle f_{n} ; n \in \mathbb{N}_{+}\right\rangle$, элементы которой определяются формулой

$$
f_{n}\left(X_{n}\right)=\sum_{\Gamma \subset I_{n}} f_{|\Gamma|}^{(1)}(X(\Gamma)) f_{n-|\Gamma|}^{(2)}\left(X\left(I_{n} \backslash \Gamma\right)\right), \quad n \in \mathbb{N}_{+},
$$

где $X(\Gamma)=\left\langle x_{j_{1}}, \ldots, x_{j_{s}}\right\rangle$ с $\Gamma=\left\{j_{1}, \ldots, x_{s}\right\}, s=|\Gamma|$. Будем считать, что $\mathrm{f}$ является результатом применения бинарной операции, обозначаемой нами далее посредством $*$, к упорядоченной паре $\left\langle\mathrm{f}^{(1)}, \mathrm{f}^{(2)}\right\rangle$ из $\mathbb{L}_{\infty}(\Omega)$.

Легко проверяется, что операция * коммутативна и ассоциативна. Она, кроме того, она является дистрибутивной по отношению к сложению элементов в $\mathbb{L}_{\infty}(\Omega)$ и билинейна по отношению к умножению элементов $f \in \mathbb{L}_{\infty}(\Omega)$ на число из $\mathbb{C}$. В связи с этим, мы будем называть ее умножением на $\mathbb{L}_{\infty}(\Omega)$. Линейное многообразие, снабженное операцией умножения $*$, превращается в алгебру над полем $\mathbb{C}$, которую мы будем обозначать тем же символом $\mathbb{L}_{\infty}(\Omega)$. В $\mathbb{L}_{\infty}(\Omega)$ имеется нейтральный элемент - последовательность $\mathrm{e}=\left\langle\delta_{n, 0} ; n \in \mathbb{N}_{+}\right\rangle$. Кроме того, в $\mathbb{L}_{\infty}(\Omega)$ всякий элемент $\mathrm{f}$, у которого $f_{0} \neq 0$ имеет обратный элемент, который мы обозначаем $\mathrm{f}_{*}^{-1}$, так, что $\mathrm{f} * \mathrm{f}_{*}^{-1}=\mathrm{e}$, т.е. в алгебре определена операция деления на элементы $f_{0} \neq 0$. По этой же причине множество 
элементов $\mathbb{L}_{\infty}^{(0)}(\Omega)=\left\{f \in \mathbb{L}_{\infty}(\Omega): f_{0}=0\right\}$, которое является подалгеброй в $\mathbb{L}_{\infty}(\Omega)$ представляет собой максимальный идеал в $\mathbb{L}_{\infty}(\Omega)$ (см. [1]). Справедливо следующее легко доказываемое утверждение.

Лемма 3.1. Для любого элемента $\mathrm{f} \in \mathbb{L}_{\infty}^{(0)}(\Omega)$ при $n<l$ имеет место равенство $\left(\mathrm{f}_{*}^{l}\right)_{n}\left(X_{n}\right)=$ 0 , а при $n \geqslant l$ справедлива следующая формула

$$
\left(\mathrm{f}_{*}^{l}\right)_{n}\left(X_{n}\right)=l ! \sum_{\mathcal{A}=\left\{\Gamma_{1}, \ldots, \Gamma_{l}\right\} \in \mathfrak{S}_{n}^{(l)}} \prod_{j=1}^{l} \mathrm{f}_{\left|\Gamma_{j}\right|}\left(X\left(\Gamma_{j}\right)\right) .
$$

Так как элементами алгебры $\mathbb{L}_{\infty}(\Omega)$ являются функции со значениями из $\mathbb{C}$, то в $\mathbb{L}_{\infty}(\Omega)$ допустимо рассмотрение степенных рядов, в частности, экспоненциальная функция:

$$
\exp _{*} \mathrm{f}=\sum_{l=0}^{\infty} \frac{1}{l !} \mathrm{f}_{*}^{l}
$$

где $\mathrm{f}_{*}^{0} \equiv$ е. Из леммы 3.1 вытекает следующее утверждение.

Лемма 3.2. Для любого элемента $\mathrm{f} \in \mathbb{L}_{\infty}^{(0)}(\Omega)$ имеет место формула

$$
\left(\exp _{*} f\right)_{n}\left(X_{n}\right)=\sum_{\mathcal{A}=\left\{\Gamma_{1}, \ldots, \Gamma_{|\mathcal{A}|}\right\} \in \mathfrak{S}_{n}} \prod_{j=1}^{|\mathcal{A}|} f_{\left|\Gamma_{j}\right|}\left(X\left(\Gamma_{j}\right)\right) .
$$

Введем линейные операторы $\partial_{x}, x \in \Omega$, на $\mathbb{L}_{\infty}(\Omega)$ посредством следующей формулы:

$$
\left(\partial_{x} \mathrm{f}\right)_{n}\left(X_{n}\right)=f_{n+1}\left(x, X_{n}\right) .
$$

Непосредственно проверяется следующее утверждение.

Лемма 3.3. Каждый оператор $\partial_{x}$ является оператором дифференцирования, т.е. для любой пары элементов $\mathrm{f} u \mathrm{~g}$ из $\mathbb{L}_{\infty}(\Omega)$ имеет место тождество Лейбница

$$
\partial_{x}(\mathrm{f} * \mathrm{~g})=\left(\partial_{x} \mathrm{f}\right) * \mathrm{~g}+\mathrm{f} *\left(\partial_{x} \mathrm{~g}\right) .
$$

Следствие 3.1. Для любого $x \in \Omega$ и любого элемента $\mathrm{f} \in \mathbb{L}_{\infty}^{(0)}(\Omega)$ имеет место формула

$$
\partial_{x} \exp _{*} f=\left(\partial_{x} f\right) * \exp _{*} f .
$$

Далее, будем полагать, что на множестве $\Omega$ имеется структура измеримости, на которой определена конечная мера $\mu$. Тогда, вводя для каждого значения $n \in \mathbb{N}$ произведение мер $d \mu\left(x_{1}\right) d \mu\left(x_{2}\right) \ldots d \mu\left(x_{n}\right)$ на $\Omega^{n}$ и ограничивая каждое из функциональных пространств $\mathbb{L}_{n}(\Omega)$ только измеримыми суммируемыми на $\Omega^{n}$ функциями $f_{n}\left(X_{n}\right)$, определим для каждой измеримой ограниченной функции $\zeta(x)$ на $\Omega$ линейный функционал на $\mathbb{L}_{n}(\Omega)$ :

$$
\mathrm{f}_{n}\left[\zeta ; f_{n}\right]=\int_{\Omega^{n}}\left(\prod_{j=1}^{n} \zeta\left(x_{j}\right)\right) f_{n}\left(X_{n}\right) d \mu\left(x_{1}\right) \ldots d \mu\left(x_{n}\right) .
$$

Рассмотрим такое сужение многообразия $\mathbb{L}_{\infty}(\Omega)$, обозначаемое далее тем же символом, в котором содержатся только элементы $\mathrm{f}=\left\langle f_{n} \in \mathbb{L}_{n}(\Omega) ; n \in \mathbb{N}_{+}\right\rangle \in \mathbb{L}_{\infty}(\Omega)$ с такими суммируемыми на $\Omega^{n}$ компонентами $f_{n}, n \in \mathbb{N}$, что сходится ряд

$$
\sum_{n=0}^{\infty} \frac{1}{n !} M^{n} \int_{\Omega^{n}}\left|f_{n}\left(X_{n}\right)\right| d \mu\left(x_{1}\right) \ldots d \mu\left(x_{n}\right)<\infty, \quad M>0 .
$$

Если функция $\zeta(x)$ ограничена постоянной $M>0,|\zeta(x)|<M, x \in \Omega$. Для таких элементов $\mathrm{f}$ определено значение функционала

$$
\mathrm{f}[\zeta ; \mathrm{f}]=\sum_{n=0}^{\infty} \frac{1}{n !} \mathrm{f}_{n}\left[\zeta ; f_{n}\right]
$$


Этот функционал обладает свойством мультипликативности, а именно, справедлива следующая теорема.

Теорема 3.1. Если элементы $\mathrm{f}^{(1)}$ и $\mathrm{f}^{(2)}$ обладают свойством (3.2) с функиией $\zeta(x)$, для которой имеет место $|\zeta(x)|<M$, то их произведение $\mathrm{f}_{1} * \mathrm{f}_{2}$ также обладает свойством (3.2) $u$ имеет место формула

$$
\mathrm{f}\left[\zeta ; \mathrm{f}^{(1)} * \mathrm{f}^{(2)}\right]=\mathrm{f}\left[\zeta ; \mathrm{f}^{(1)}\right] \cdot \mathrm{f}\left[\zeta ; \mathrm{f}^{(2)}\right] .
$$

Доказательство осуществляется прямым вычислением:

$$
\begin{aligned}
& \mathrm{f}_{n}\left[\zeta ; \mathrm{f}^{(1)} * \mathrm{f}^{(2)}\right]=\int_{\Omega^{n}}\left(\prod_{j=1}^{n} \zeta\left(x_{j}\right)\right)\left(\mathrm{f}^{(1)} * \mathrm{f}^{(2)}\right)_{n}\left(X_{n}\right) d \mu\left(x_{1}\right) \ldots d \mu\left(x_{n}\right)= \\
&=\sum_{l=0}^{n}\left(\begin{array}{c}
n \\
l
\end{array}\right) \int_{\Omega^{n}}\left(\prod_{j=1}^{n} \zeta\left(x_{j}\right)\right) f_{l}^{(1)}\left(X_{l}\right) f_{n-l}^{(2)}\left(X\left(I_{n} \backslash I_{l}\right)\right) d \mu\left(x_{1}\right) \ldots d \mu\left(x_{n}\right)= \\
& \quad=\sum_{l=0}^{n}\left(\begin{array}{c}
n \\
l
\end{array}\right) \mathrm{f}_{l}\left[\zeta ; f_{l}^{(1)}\right] \cdot \mathrm{f}_{n-l}\left[\zeta ; f_{n-l}^{(2)}\right] .
\end{aligned}
$$

Подставляя полученное выражение в $\mathrm{f}\left[\zeta ; \mathrm{f}^{(1)} * \mathrm{f}^{(2)}\right]$, находим

$$
\mathrm{f}\left[\zeta ; \mathrm{f}^{(1)} * \mathrm{f}^{(2)}\right]=\sum_{n=0}^{\infty} \frac{1}{n !} \sum_{l=0}^{n}\left(\begin{array}{l}
n \\
l
\end{array}\right) \mathrm{f}_{l}\left[\zeta ; f_{l}^{(1)}\right] \cdot \mathrm{f}_{n-l}\left[\zeta ; f_{n-l}^{(2)}\right]=\mathrm{f}\left[\zeta ; \mathrm{f}^{(1)}\right] \cdot \mathrm{f}\left[\zeta ; \mathrm{f}^{(2)}\right] .
$$

Следствие 3.2. Справедлива формула

$$
\mathrm{f}\left[\zeta ; \exp _{*} \mathrm{f}\right]=\exp \mathrm{f}[\zeta ; \mathrm{f}] .
$$

Следствие 3.3. Имеет место формула дифференцирования

$$
\mathrm{f}\left[\zeta ; \partial_{x} \exp _{*} \mathrm{f}\right]=\mathrm{f}\left[\zeta ; \partial_{x} \mathrm{f}\right] \cdot \exp \mathrm{f}[\zeta ; \mathrm{f}] .
$$

4. Графы и симметрические функции. Пусть $w(x, y)$-произвольная симметрическая функция $\Omega^{2}$, которую мы будем называть порождающей. Зафиксируем $n \in \mathbb{N}, n \geqslant 2$, и поставим в соответствие паре $\left\langle w \in \mathbb{L}_{2}(\Omega), \mathfrak{G}=\left\langle I_{n}, \Psi\right\rangle\right\rangle$ функцию на $\Omega^{n}$, определяемую формулой

$$
h_{n}\left(X_{n} ; \mathfrak{G}\right)=\prod_{\{i, j\} \in \Psi} w\left(x_{i}, x_{j}\right) .
$$

Каждую такую функцию будем называть функиией на $\Omega^{n}$, ассоииированной с графом $\mathfrak{G}$ посредством порождающей функции $w$.

На основе функций $h_{n}(\cdot ; \mathfrak{G})$, ассоциированных с графами, посредством следующей конструкции, строятся симметрические функции, которые являются элементами пространства $\mathbb{L}_{n}(\Omega)$. Зафиксируем какой-либо класс $\mathcal{H}$ графов над $I_{n}$, который обладает свойством инвариантности относительно перестановок, так что для $\mathrm{P} \in \mathbb{P}_{n}$ и $\mathfrak{G} \in \mathcal{H}$ имеем $\mathrm{P} \mathfrak{G}=\left\langle I_{n}, \mathrm{P} \Psi\right\rangle \in \mathcal{H}$. Определим функцию

$$
f_{n}\left(X_{n}\right)=\sum_{\mathfrak{G} \in \mathcal{H}} h_{n}\left(X_{n} ; \mathfrak{G}\right)
$$

на $\Omega^{n}$, которая, очевидно, является симметрической. Каждую функцию $f_{n}\left(X_{n}\right)$ на $\Omega^{n}$, построенную согласно (4.1), будем называть ассоциированной с классом $\mathcal{H}$. Возьмем в качестве $\mathcal{H}$ класс $\mathcal{G}_{n}$ всех графов над $I_{n}$.

Лемма 4.1. Пусть $w(x, y)$-симметрическая функиия на $\Omega^{2}$. Тогда функиия, ассоциированная с классом $\mathcal{G}_{n}$ на основе порождающей функции $w(x, y)$, равна

$$
f_{n}\left(X_{n}\right)=\sum_{\mathfrak{G}=\left\langle I_{n} ; \Psi\right\rangle \in \mathcal{G}_{n}} \prod_{\{i, j\} \in \Psi} w\left(x_{i}, x_{j}\right)=\prod_{\{i, j\} \in I_{n}^{(2)}}\left(1+w\left(x_{i}, x_{j}\right)\right) .
$$


Доказательство проводится индукцией по $n$ на основе формулы

$$
\begin{aligned}
\sum_{\mathfrak{G}=\left\langle I_{n+1} ; \Psi\right\rangle \in \mathcal{G}_{n+1}} \prod_{\{i, j\} \in \Psi} w\left(x_{i}, x_{j}\right)= & \sum_{\mathfrak{G}=\left\langle I_{n} ; \Psi\right\rangle \in \mathcal{G}_{n}} \prod_{\{k, l\} \in \Psi} w\left(x_{k}, x_{l}\right)+ \\
& +\sum_{\mathfrak{G}=\left\langle I_{n} ; \Psi\right\rangle \in \mathcal{G}_{n}} \sum_{\Gamma \subset I_{n}} \prod_{j \in \Gamma} w\left(x_{n+1}, x_{j}\right)\left[\prod_{\{k, l\} \in \Psi} w\left(x_{k}, x_{l}\right)\right],
\end{aligned}
$$

обеспечивающей индукционный шаг. Здесь первая сумма соответствует графам, у которых вершина $n+1$ не связана ни с одной вершиной из $I_{n}$, а вторая сумма учитывает все графы класса $\mathcal{G}_{n+1}$, у которых вершина $n+1$ связывается с вершинами графов класса $\mathcal{G}_{n}$, множество номеров которых составляют $Г$.

Заметим, что класс $\mathcal{G}_{n}$ всех графов над $I_{n}$ инвариантен относительно перестановок $\mathrm{P} \in \mathbb{P}_{n}$, и поэтому при любом $n \in \mathbb{N}$ функции $f_{n}$, ассоциированные с $\mathcal{G}_{n}$, являются симметрическими, т.е. принадлежат $\mathbb{L}_{n}(\Omega)$.

Наряду с функциями $f_{n}\left(X_{n}\right)$, ассоциированными с классами $\mathcal{G}_{n}, n \in \mathbb{N}, n \geqslant 2$, введем последовательность функций $\bar{f}_{n}\left(X_{n}\right)$ с порождающей функцией $w(x, y)$, каждая из которых ассоциирована с классом $\overline{\mathcal{G}}_{n}$ всех связных графов на $I_{n}, n \geqslant 2$ :

$$
\bar{f}_{n}\left(X_{n}\right)=\sum_{\mathfrak{G}=\left\langle I_{n} ; \Psi\right\rangle \in \overline{\mathcal{G}}_{n}} \prod_{\{i, j\} \in \Psi} w\left(x_{i}, x_{j}\right) .
$$

Так как при каждом $n \in \mathbb{N}$ класс $\overline{\mathcal{G}}_{n}$ инвариантен относительно перестановок $\mathrm{P} \in \mathbb{P}_{n}$, то функции $\bar{f}_{n}\left(X_{n}\right)$ при каждом $n \geqslant 2$ являются симметрическими. Таким образом, функции $f_{n}$ и $\bar{f}_{n}$ принадлежат $\mathbb{L}_{n}(\Omega)$ при любом $n \geqslant 2$. Докажем, что для составленных последовательностей функций из $\mathbb{L}_{\infty}(\Omega)$ справедлива следующая теорема.

Теорема 4.1. Пусть элементы $\mathrm{f}=\left\langle f_{n} ; n \in \mathbb{N}_{+}\right\rangle u \overline{\mathrm{f}}=\left\langle\bar{f}_{n} ; n \in \mathbb{N}_{+}\right\rangle$алгебры $\mathbb{L}_{\infty}(\Omega)$, компоненты которых при $n \geqslant 2$ определяются формулами (4.2) и (4.3) соответственно на основе одной и той же порождающей функиии $w(x, y) \in \mathbb{L}_{2}(\Omega)$ и при этом $f_{0}=1, \bar{f}_{0}=0, \bar{f}_{1}=f_{1}=1$, то эти элементы связаны следующим образом:

$$
\mathrm{f}=\exp _{*} \overline{\mathrm{f}} .
$$

Доказательство. Докажем, что для функций $f_{n}$ имеет место соотношение

$$
f_{n}\left(X_{n}\right)=\sum_{\mathcal{A} \in \mathfrak{S}_{n}} \prod_{\Gamma \in \mathcal{A}} \bar{f}_{|\Gamma|}(X(\Gamma))
$$

Распределим следующим образом все графы класса $\mathcal{G}_{n}$ на непересекающиеся классы $\mathcal{G}_{n}(\mathcal{A})$, где $\mathcal{A}=\left\{\Gamma_{1}, \ldots, \Gamma_{s}\right\} \in \mathfrak{S}_{n}$. Рассмотрим произвольный граф $\mathfrak{G}=\left\langle I_{n}, \Psi\right\rangle$ из $\mathcal{G}_{n}$. Этот граф распадается однозначным образом на связанные компоненты $\mathfrak{G}_{j}=\left\langle\Gamma_{j}, \Psi_{j}\right\rangle, j=1, \ldots, s$, так, что

$$
\bigcup_{j=1}^{s} \Gamma_{j}=I_{n}, \quad \bigcup_{j=1}^{n} \Psi_{j}=\Psi
$$

и каждый из графов $\mathfrak{G}_{j}$ принадлежит классу $\overline{\mathcal{G}}_{\left|\Gamma_{j}\right|}\left(\Gamma_{j}\right)$ всех связных графов над множеством вершин $\Gamma_{j}$. В этом случае отнесем граф $\mathfrak{G}$ к классу $\mathcal{G}_{n}(\mathcal{A}), \mathcal{A}=\left\{\Gamma_{1}, \ldots, \Gamma_{|\mathcal{A}|}\right\}$. Ясно, что классы $\mathcal{G}_{n}\left(\mathcal{A}_{1}\right)$ и $\mathcal{G}_{n}\left(\mathcal{A}_{2}\right)$ не пересекаются, если $\mathcal{A}_{1} \neq \mathcal{A}_{2}$, и не пусты. Тогда имеет место следующее представление суммы по всем графам класса $\mathcal{G}_{n}$ :

$$
\sum_{\mathfrak{G} \in \mathcal{G}_{n}} \cdots=\sum_{\mathcal{A} \in \mathfrak{S}_{n}}\left(\prod_{j=1}^{|\mathcal{A}|} \sum_{\left.\mathfrak{G}_{j} \in \mathcal{G}_{\left|\Gamma_{j}\right|} \mid \Gamma_{j}\right)}\right) \ldots
$$


При этом для каждого слагаемого суммы имеет место соотношение

$$
\prod_{\{k, l\} \in \Psi} w\left(x_{k}, x_{l}\right)=\prod_{j=1}^{|\mathcal{A}|} \prod_{\{k, l\} \in \Psi_{j}} w\left(x_{k}, x_{l}\right),
$$

ввиду несвязанности графов $\mathfrak{G}_{j}, j=1, \ldots,|\mathfrak{D}|$. Подставляя произведение в правой части в сумму, определяющую $f_{n}\left(X_{n}\right)$, находим

$$
\begin{aligned}
f_{n}\left(X_{n}\right)=\sum_{\mathfrak{G} \in \mathcal{G}_{n}} \prod_{\{k, l\} \in \Psi} w\left(x_{k}, x_{l}\right)= \\
\quad=\sum_{\mathcal{A} \in \mathfrak{S}_{n}} \prod_{j=1}^{|\mathcal{A}|}\left(\sum_{\left.\mathfrak{G}_{j} \in \mathcal{G}_{\left|\Gamma_{j}\right|} \mid \Gamma_{j}\right)} \prod_{\{k, l\} \in \Psi_{j}} w\left(x_{k}, x_{l}\right)\right)=\sum_{\mathcal{A} \in \mathfrak{S}_{n}}\left(\prod_{j=1}^{|\mathcal{A}|} \bar{f}_{\left|\Gamma_{j}\right|}\left(X\left(\Gamma_{j}\right)\right)\right) .
\end{aligned}
$$

Применяя формулу (3.3), из этой теоремы получаем следующий результат.

Следствие 4.1. Если на множестве $\Omega$ определена структура измеримости с конечной мерой и ограниченная измеримая функиия $\zeta$ на $\Omega$, то для значений функционала $\mathrm{f}[\zeta ; \cdot]$ на суммируемых элементах $\mathrm{f} \in \mathbb{L}_{\infty}(\Omega), \overline{\mathrm{f}} \in \mathbb{L}_{\infty}^{(0)}(\Omega)$, построеннных на основе фиксированной порождающей функиии $w$, имеет место формула

$$
\mathrm{f}[\zeta, \mathrm{f}]=\exp \mathrm{f}[\zeta ; \overline{\mathrm{f}}] .
$$

Заметим, что это утверждение остается справедливым и в том случае, когда ряды, определяющие указанные значения функционала расходятся.

5. Симметрические функции и графы класса $\mathcal{F}_{n}$. Пусть на множестве $\Omega$ определена группа $\mathbb{T}$ преобразований Т, причем для любого $x \in \Omega$ имеет место $\Omega=\{\mathrm{T} x ; \boldsymbol{\top} \in \mathbb{T}\}$, т.е. $\Omega$ инвариантно относительно преобразований этой группы. В этом разделе мы получим основной результат работы: уравнение, связывающее значения функционала $\mathrm{f}[z ; \overline{\mathrm{f}}]$ и $\mathrm{f}[z ; \mathrm{g}] \mathrm{c} \zeta(x) \equiv z \in \mathbb{C}$, где функции $\mathrm{g}=\left\langle g_{n} ; n \in \mathbb{N}_{+}\right\rangle$ассоциированы со связными графами без вершин сочленения и, вместе с функциями последовательности $\overline{\mathfrak{f}}$, порождаются симметрической функцией $w(x, y)$, инвариантной относительно преобразований $\mathrm{T} \in \mathbb{T}$.

Рассмотрим подалгебру $\mathbb{L}_{+}(\Omega)$ таких элементов $\mathrm{h}$ алгебры $\mathbb{L}_{\infty}(\zeta)$, у которых все их компоненты $h_{n}\left(X_{n}\right), n \in \mathbb{N}_{+}$, инвариантны относительно преобразований группы $\mathbb{T}$ ( $\mathbb{T}$-инвариантны). $\mathrm{B}$ частности, для значения $n=1$ линейное многообразие $\mathbb{L}_{1}$ в этой алгебре совпадает с $\mathbb{C}$.

Кроме того, потребуем, чтобы все компоненты каждой последовательности из $\mathbb{L}_{+}(\Omega)$ являлись суммируемыми в следующем смысле с $\mathbb{T}$-инвариантной мерой $\mu$

$$
\int_{\Omega^{n-1}}\left|h_{n}\left(X_{n}\right)\right| \prod_{j=1}^{n-1} d \mu\left(x_{j}\right)<\infty,
$$

а вся их совокупность обладает тем свойством, что для элементов $\mathrm{h}$ из максимального идеала $\mathbb{L}_{+}^{(0)}(\Omega)=\mathbb{L}_{+}(\Omega) \cap \mathbb{L}_{\infty}^{(0)}(\Omega)$ алгебры $\mathbb{L}_{+}(\Omega)$ имеется достаточно малая окрестность точки $z=0$ в плоскости $z \in \mathbb{C}$, в которой сходится степенной ряд

$$
\sum_{n=1}^{\infty} \frac{z^{n-1}}{(n-1) !} \int_{\Omega^{n-1}}\left|h_{n}\left(X_{n}\right)\right| \prod_{j=1}^{n-1} d \mu\left(x_{j}\right)<\infty .
$$

На элементах $\mathrm{h}=\left\langle h_{n}\left(X_{n}\right) ; n \in \mathbb{N}_{+}\right\rangle \in \mathbb{L}_{+}^{(0)}(\Omega)$ рассмотрим функционал

$$
\mathrm{S}[z ; \mathrm{h}]=\mathrm{f}\left[z ; \partial_{x} \mathrm{~h}\right]=\sum_{n=0}^{\infty} \frac{z^{n}}{n !} \mathrm{f}_{n}\left[h_{n+1}\right],
$$


где функционалы $\mathrm{f}_{n}[\cdot]$ при $n \in \mathbb{N}$ определяются $\mathbb{T}$-инвариантной мерой $\mu$ и весовой функцией $\zeta=1$ в соответствии с (3.1),

$$
\mathrm{f}_{n}\left[h_{n+1}\right]=\int_{\Omega^{n}} h_{n+1}\left(X_{n+1}\right) \prod_{j=1}^{n} d \mu\left(x_{j}\right) \quad \text { и } \quad \mathrm{f}_{0}\left[h_{1}\right]=h_{1} .
$$

Для доказательства основного утверждения этого приложения нам понадобится следующее простое комбинаторное утверждение.

Лемма 5.1. Пусть $\varphi\left(\xi_{1}, \ldots, \xi_{s}\right)$ - произвольная функиия на $\mathbb{N}^{s}$. Тогда при $n \geqslant s$ имеет место формула

$$
\sum_{\substack{\left\langle A_{1}, \ldots, A_{s}\right\rangle: \\ A_{j} \neq \varnothing, A_{j} \subset I_{n}, j=1, \ldots, s ; \\ A_{j} \cap A_{k}=\varnothing, j \neq k ; \bigcup_{j=1}^{s} A_{j}=I_{n}}} \varphi\left(\left|A_{1}\right|, \ldots,\left|A_{s}\right|\right)=\sum_{\substack{\left\langle l_{1}, \ldots, l_{s}\right\rangle: l_{j} \geqslant 1 ; \\ l_{1}+\cdots+l_{s}=n}} \frac{n !}{l_{1} ! \ldots l_{s} !} \varphi\left(l_{1}, \ldots, l_{s}\right) .
$$

Доказательство проводится индукцией по $s$. При $s=1$ сумма в (5.1) состоит из одного слагаемого и формула превращается в тождество:

$$
\sum_{A_{1}=I_{n}} \varphi\left(\left|A_{1}\right|\right)=\sum_{l_{1}=n} \frac{n !}{l_{1} !} \varphi\left(l_{1}\right) .
$$

Индукционный шаг строится следующим образом:

$$
\begin{gathered}
\sum_{\substack{\left\langle A_{1}, \ldots, A_{s+1}\right\rangle: \\
A_{j} \neq \varnothing, A_{j} \subset I_{n}, j=1, \ldots, s+1 ; \\
A_{j} \cap A_{k}=\varnothing, j \neq k ; \bigcup_{j=1}^{s+1} A_{j}=I_{n}}} \varphi\left(\left|A_{1}\right|, \ldots,\left|A_{s+1}\right|\right)= \\
=\sum_{\substack{\varnothing \neq A_{s+1} \subset I_{n}: \\
\left|A_{s+1}\right|=1, \ldots, n-s}} \sum_{\substack{\left\langle A_{1}, \ldots, A_{s}\right\rangle: \\
A_{j} \neq \varnothing, A_{j} \subset I_{n} \backslash A_{s+1}, j=1, \ldots, s ; \\
A_{j} \cap A_{k}=\varnothing, j \neq k ; \bigcup_{j=1}^{s} A_{j}=I_{n} \backslash A_{s+1}}} \varphi\left(\left|A_{1}\right|, \ldots,\left|A_{s+1}\right|\right)= \\
=\sum_{l_{s+1}=1}^{n-s}\left(\begin{array}{c}
n \\
l_{s+1}
\end{array}\right) \sum_{\substack{\left\langle A_{1}, \ldots, A_{s}\right\rangle: \\
A_{j} \neq \varnothing, A_{j} \cap A_{k}=\varnothing, I_{n} \backslash A_{s+1}, j=k ; \bigcup_{j=1}^{s} A_{j}=I_{n} \backslash A_{s+1}}} \varphi\left(\left|A_{1}\right|, \ldots,\left|A_{s}\right|, l_{s+1}\right) .
\end{gathered}
$$

Используя предположение индукции для внутренней суммы, запишем

$$
\begin{aligned}
\sum_{l_{s+1}=1}^{n-s}\left(\begin{array}{c}
n \\
l_{s+1}
\end{array}\right) \sum_{\substack{\left\langle l_{1}, \ldots, l_{s}\right\rangle: l_{j} \geqslant 1 ; \\
l_{1}+\cdots+l_{s}=n-l_{s+1}}} \frac{\left(n-l_{s+1}\right) !}{l_{1} ! \ldots l_{s} !} \varphi\left(l_{1}, \ldots, l_{s}, l_{s+1}\right) & \\
& =\sum_{\substack{\left\langle l_{1}, \ldots, l_{s+1}\right\rangle: l_{j} \geqslant 1 ; \\
l_{1}+\cdots+l_{s+1}=n}} \frac{n !}{l_{1} ! \ldots l_{s+1} !} \varphi\left(l_{1}, \ldots, l_{s}, l_{s+1}\right) .
\end{aligned}
$$


Следствие 5.1. Пусть $\varphi\left(\xi_{1}, \ldots, \xi_{s}\right)$ - произвольная функция на $\mathbb{N}^{s}$. Тогда для суммирования по разложениям $\mathcal{A}=\left\{A_{1}, \ldots, A_{s}\right\} \in \mathfrak{S}_{n}^{(s)}$ множества $I_{n}$ при $n \geqslant s$ имеет место формула

$$
\sum_{\substack{\left\{A_{1}, \ldots, A_{s}\right\}: \\ A_{j} \neq \varnothing, A_{j} \subset I_{n}, j=1, \ldots, s ; \\ A_{j} \cap A_{k}=\varnothing, j \neq k ; \bigcup_{j=1}^{s} A_{j}=I_{n}}} \varphi\left(\left|A_{1}\right|, \ldots,\left|A_{s}\right|\right)=\frac{1}{s !} \sum_{\substack{\left\langle l_{1}, \ldots, l_{s}\right\rangle: l_{j} \geqslant 1 ; \\ l_{1}+\cdots+l_{s}=n}} \frac{n !}{l_{1} ! \ldots l_{s} !} \varphi\left(l_{1}, \ldots, l_{s}\right) .
$$

Доказательство следует из того, что каждое разложение $\mathcal{A}=\left\{A_{1}, \ldots, A_{s}\right\} \in \mathfrak{S}_{n}^{(s)}$ множества $I_{n}$ порождает ровно $s$ ! упорядоченных наборов $\left\langle A_{1}, \ldots, A_{s}\right\rangle$.

Рассмотрим функции

$$
\bar{f}_{n+1}\left(X_{n+1}\right)=\sum_{\mathfrak{G} \in \overline{\mathcal{G}}_{n}} h\left(X_{n+1} ; \mathfrak{G}\right), \quad h\left(X_{n+1} ; \mathfrak{G}\right)=\prod_{\{j, k\} \in \Psi} w\left(x_{j}, x_{k}\right),
$$

где $h\left(X_{n+1} ; \mathfrak{G}\right)$ на $\Omega^{n+1}$ ассоциированы с графами $\mathfrak{G}=\left\langle I_{n+1}, \Psi\right\rangle \in \overline{\mathcal{G}}_{n}$ посредством $\mathbb{T}$-инваринатной порождающей функции $w(x, y)$ на $\Omega^{2}$. При этом $\bar{f}=\left\langle\bar{f}_{n+1} ; n \in \mathbb{N}_{+}\right\rangle \in \mathbb{L}_{+}^{(0)}(\Omega)$. Точно так же введем функции

$$
g_{n+1}\left(X_{n+1}\right)=\sum_{\mathfrak{G} \in \mathcal{F}\left[I_{n+1}\right]} h\left(X_{n+1} ; \mathfrak{G}\right), \quad n \in \mathbb{N}_{+},
$$

где $\mathcal{F}\left[I_{n+1}\right]$ - класс графов без вершин сочленения над $I_{n+1}$, которые составляют элемент $\mathrm{g}=$ $\left\langle g_{n+1} ; n \in \mathbb{N}_{+}\right\rangle$алгебры $\mathbb{L}_{+}(\Omega)$. Сформулируем теперь основной результат работы.

Теорема 5.1. Значения $\mathrm{S}[z ; \overline{\mathrm{f}}]$ u $\mathrm{S}[z ; \mathrm{g}]$ функиионала $\mathrm{S}[z ; \cdot]$ на элементах $\overline{\mathrm{f}} u \mathrm{~g}$ подчинены следующему функииональному уравнению:

$$
\mathrm{S}[z ; \overline{\mathrm{f}}]=\exp (\mathrm{S}[z \mathrm{~S}[z ; \bar{f}] ; \mathrm{g}]-1) .
$$

\section{Доказательство.}

1. Согласно лемме 2.1 , так как классы $\overline{\mathcal{G}}_{n+1}\left[I_{n} ; n+1\right], n \in \mathbb{N}$, представимы в виде дизъюнктивных объединений

$$
\overline{\mathcal{G}}\left[I_{n}, n+1\right]=\bigcup_{s=1}^{n} \overline{\mathcal{G}}^{(s)}\left[I_{n} ; n+1\right]
$$

где отмеченная вершина сочленения $n+1$ имеет степень $s$, то каждая из функций $\bar{f}_{n+1}\left(X_{n+1}\right)$ представима в виде суммы

$$
\bar{f}_{n+1}\left(X_{n+1}\right)=\sum_{s=1}^{n} f_{n+1}^{(s)}\left(X_{n+1}\right), \quad f_{n+1}^{(s)}\left(X_{n+1}\right)=\sum_{\mathfrak{G} \in \overline{\mathcal{G}}^{(s)}\left[I_{n}, n+1\right]} h\left(X_{n+1} ; \mathfrak{G}\right) .
$$

При этом ввиду симметрии классов $\overline{\mathcal{G}}^{(s)}\left[I_{n}, n+1\right]$ относительно перестановок $\mathrm{P} \in \mathbb{P}_{n}$ и $\mathbb{T}$-инвариантности $w(x, y)$, функции $f_{n+1}^{(s)}\left(X_{n+1}\right)$ являются симметрическими относительно таких $\mathrm{P}$ и $\mathbb{T}$ инвариантны. Кроме того, имеет место равенство

$$
\mathrm{f}_{n}\left[\bar{f}_{n+1}\right]=\sum_{s=1}^{n} \mathrm{f}_{n}\left[f_{n+1}^{(s)}\right] .
$$

2. Далее, согласно лемме 2.2 , если вершина $n+1$ имеет степень сочленения $s>1$, то класс $\overline{\mathcal{G}}^{(s)}\left[I_{n} ; n+1\right]$ представим в виде следующего дизъюнктивного объединения

$$
\overline{\mathcal{G}}^{(s)}\left[I_{n} ; n+1\right]=\bigcup_{\mathcal{A} \in \mathfrak{S}_{n}^{(s)}} \overline{\mathcal{G}}^{(s)}\left[I_{n} ; \mathcal{A}, n+1\right],
$$

где у связанных графов из $\overline{\mathcal{G}}^{(s)}\left[I_{n} ; \mathcal{A}, n+1\right]$ над $I_{n+1}$ вершина $n+1$ имеет степень сочленения $s$, а номера вершин, отличных от вершины $n+1$, у связных графов $\mathfrak{G}_{j}, j=1, \ldots, s$, составляют 
разложение $\mathcal{A} \in \mathfrak{S}_{n}^{(s)}$ с числом компонент $s$ и $n+1$ не является у них вершиной сочленения. Тогда функции $f_{n+1}^{(s)}$ представимы в виде сумм

$$
f_{n+1}^{(s)}\left(X_{n+1}\right)=\sum_{\mathcal{A} \in \mathfrak{S}_{n}^{(s)}} f_{n+1}^{(s)}\left(X_{n+1} ; \mathcal{A}, n+1\right),
$$

где функции $f_{n+1}^{(s)}\left(X_{n+1} ; \mathcal{A}, n+1\right)$ определяются формулой

$$
f_{n+1}^{(s)}\left(X_{n+1} ; \mathcal{A}, n+1\right)=\sum_{\mathfrak{G} \in \overline{\mathcal{G}}^{(s)}\left[I_{n} ; \mathcal{A}, n+1\right]} h\left(X_{n+1} ; \mathfrak{G}\right) .
$$

Они симметричны относительно всех $\mathrm{P} \in \mathbb{P}_{n}$, которые оставляют инвариантными компоненты разложения $\mathcal{A}$. При этом имеет место

$$
\mathrm{f}_{n}\left[f_{n+1}^{(s)}\right]=\sum_{\mathcal{A} \in \mathfrak{S}_{n}^{(s)}} \mathrm{f}_{n}\left[f_{n+1}^{(s)}\left(X_{n+1} ; \mathcal{A}, n+1\right)\right] .
$$

3. Согласно лемме 2.3 , для любого разложения $\mathcal{A}=\left\{A_{1}, \ldots, A_{s}\right\} \in \mathfrak{S}_{n}^{(s)}$ класс $\overline{\mathcal{G}}^{(s)}\left[I_{n} ; \mathcal{A}, n+1\right]$, $n \geqslant s>1$, эквивалентен декартову произведению

$$
\overline{\mathcal{G}}^{(s)}\left[I_{n} ; \mathcal{A}, n+1\right]=\bigotimes_{l=1}^{s} \overline{\mathcal{G}}^{(1)}\left[A_{l} ; n+1\right]
$$

классов $\overline{\mathcal{G}}^{(1)}\left[A_{l} ; n+1\right]$ связных графов над множеством $A_{l} \cup\{n+1\}, l=2, \ldots, s$, с выделенной вершиной $n+1$, у которых вершина $n+1$ не является вершиной сочленения. Тогда имеет место следующее представление для суммы

$$
\sum_{\mathfrak{G} \in \overline{\mathcal{G}}^{(s)}\left[I_{n} ; \mathcal{A}, n+1\right]} h\left(X_{n+1} ; \mathfrak{G}\right)=\left(\prod_{l=1}^{s} \sum_{\mathfrak{G} \in \overline{\mathcal{G}}^{(1)}\left[A_{l} ; n+1\right]}\right) h\left(X_{n+1} ; \mathfrak{G}\right) .
$$

При этом, так как для каждого графа $\mathfrak{G} \in \overline{\mathcal{G}}^{(s)}\left[I_{n} ; \mathcal{A}, n+1\right]$, где $\mathcal{A}=\left\{A_{j} ; j=1, \ldots,|\mathcal{A}|\right\}$, ассоциированная с ним симметрическая функция представима в виде

$$
h\left(X_{n+1} ; \mathfrak{G}\right)=\prod_{l=1}^{s} h\left(X\left(A_{l} \cup\{n+1\}\right) ; \mathfrak{G}_{l}\right), \quad \mathfrak{G}=\bigvee_{l=1}^{s} \mathfrak{G}_{l},
$$

Tо

$$
\begin{aligned}
\mathrm{f}_{n}\left[f\left(X_{n+1} ; \mathcal{A}\right)\right]=\left(\prod_{l=1}^{s} \sum_{\mathfrak{G} \in \overline{\mathcal{G}}^{(1)}\left[A_{l} ; n+1\right]}\right) \mathrm{f}_{n}\left[\prod_{l=1}^{s} h\left(X\left(A_{l} \cup\{n+1\}\right) ; \mathfrak{G}_{l}\right)\right]= \\
=\prod_{l=1}^{s} \mathrm{f}_{\left|A_{l}\right|}\left[\sum_{\mathfrak{G} \in \overline{\mathcal{G}}^{(1)}\left[A_{l} ; n+1\right]} h\left(X\left(A_{l} \cup\{n+1\}\right) ; \mathfrak{G}_{l}\right)\right]=\prod_{l=1}^{s} \mathrm{f}_{\left|A_{l}\right|}\left[f_{\left|A_{l}\right|+1}^{(1)}\left(X\left(A_{l} \cup\{n+1\}\right)\right)\right],
\end{aligned}
$$

где учтено, что для графа $\mathfrak{G}_{l_{1}} \vee \mathfrak{G}_{l_{2}}$ с множествами вершин $A_{l_{1}}$ и $A_{l_{2}}$ соответственно, склеенного из двух графов $\mathfrak{G}_{l_{1}}$ и $\mathfrak{G}_{l_{2}}$ в вершине $n+1$, выполняется

$$
\begin{aligned}
\mathrm{f}_{\left|A_{l_{1} \mid}\right| A_{l_{2}} \mid}\left[h\left(X\left(A_{l_{1}} \cup A_{l_{2}} \cup\{n+1\}\right) ; \mathfrak{G}_{l_{1}} \vee \mathfrak{G}_{l_{2}}\right)\right]= \\
=\sum_{X\left(A_{l_{1}} \cup A_{l_{2}}\right) \in \Omega^{\left|A_{l_{1} \mid}+\right| A_{l_{2}} \mid}} h\left(X\left(A_{l_{1}} \cup A_{l_{2}} \cup\{n+1\}\right) ; \mathfrak{G}_{l_{1}} \vee \mathfrak{G}_{l_{2}}\right)=
\end{aligned}
$$




$$
\begin{gathered}
=\left(\sum_{X\left(A_{l_{1}}\right) \in \Omega^{\left|A_{l_{1}}\right|}} h\left(X\left(A_{l_{1}} \cup\{n+1\}\right) ; \mathfrak{G}_{l_{1}}\right)\right)\left(\sum_{X\left(A_{l_{2}}\right) \in \Omega^{\left|A_{l_{2}}\right|}} h\left(X\left(A_{l_{2}} \cup\{n+1\}\right) ; \mathfrak{G}_{l_{2}}\right)\right)= \\
\left.=\mathrm{f}_{\left|A_{l_{1}}\right|}\left[h\left(X\left(A_{l_{1}} \cup\{n+1\}\right) ; \mathfrak{G}_{l_{1}}\right)\right] \cdot \mathfrak{f}_{\left|A_{l_{2}}\right|} \mid h\left(X\left(A_{l_{2}} \cup\{n+1\}\right) ; \mathfrak{G}_{l_{2}}\right)\right] .
\end{gathered}
$$

4. На основании формул (5.5), (5.6), (5.7), применяя (5.2), получаем следующее выражение для функционала $\mathrm{f}_{n}$ на функциях $\bar{f}_{n+1}\left(X_{n+1}\right)$ :

$$
\begin{aligned}
\mathrm{f}_{n}\left[\bar{f}_{n+1}\right]=\mathrm{f}_{n}\left[\bar{f}_{n+1}^{(1)}\right]+\sum_{s=2}^{n} \mathrm{f}_{n}\left[f_{n+1}^{(s)}\right]=\mathrm{f}_{n}\left[f_{n+1}^{(1)}\right]+\sum_{s=2}^{n} \sum_{\mathcal{A} \in \mathfrak{S}_{n}^{(s)}} \mathrm{f}_{n}\left[f_{n+1}^{(s)}\left(X_{n+1} ; \mathcal{A}\right)\right]= \\
=\mathrm{f}_{n}\left[\bar{f}_{n+1}^{(1)}\right]+\sum_{s=2}^{n} \sum_{\mathcal{A} \in \mathfrak{S}_{n}^{(s)}} \prod_{j=1}^{s} \mathrm{f}_{\left|A_{j}\right|}\left[\bar{f}_{\left|A_{j}\right|+1}\left(X\left(A_{j} \cup\{n+1\}\right)\right)\right]= \\
=\mathrm{f}_{n}\left[\bar{f}_{n+1}^{(1)}\right]+\sum_{s=2}^{n} \frac{1}{s !} \sum_{\substack{l_{j} \geqslant 1, j=1, \ldots, s: \\
l_{1}+\cdots+l_{s}=n}} \frac{n !}{l_{1} ! \ldots l_{s} !} \prod_{j=1}^{s} \mathrm{f}_{l_{j}}\left[\bar{f}_{l_{j}+1}^{(1)}\left(X_{l_{j}}, x_{n+1}\right)\right]= \\
=\sum_{s=1}^{n} \frac{1}{s !} \sum_{\substack{l_{j} \geqslant 1, j=1, \ldots, s: \\
l_{1}+\cdots+l_{s}=n}} \frac{n !}{l_{1} ! \ldots l_{s} !} \prod_{j=1}^{s} \mathrm{f}_{l_{j}}\left[\bar{f}_{l_{j}+1}^{(1)}\left(X_{l_{j}}, x_{n+1}\right)\right] .
\end{aligned}
$$

5. Рассмотрим теперь графы класса $\overline{\mathcal{G}}^{(1)}\left[I_{n}, n+1\right]$. Согласно лемме 2.4 , класс $\overline{\mathcal{G}}^{(1)}\left[I_{n}, n+1\right]$, $n \geqslant 2$, представим в виде дизъюнктивного объединения

$$
\overline{\mathcal{G}}^{(1)}\left[I_{n}, n+1\right]=\bigcup_{B \subset I_{n}:|B| \geqslant 1} \bigcup_{C \subset B} \overline{\mathcal{G}}\left[I_{n} ; B ; C\right]
$$

классов графов, у которых $n+1$ не является вершиной сочленения, причем для каждого графа множество $B$ представляет номера вершин того блока в нем, который содержит вершину $n+1$, а $C$-множество вершин сочленения графа в этом блоке. Тогда

$$
\begin{gathered}
f_{n+1}^{(1)}\left(X_{n+1}\right)=\sum_{B \subset I_{n}:|B| \geqslant 1} \sum_{C \subset B} f_{n+1}\left(X_{n+1} ; B ; C\right), \\
f_{n+1}\left(X_{n+1} ; B ; C\right)=\sum_{\mathfrak{G} \in \overline{\mathcal{G}}\left[I_{n} ; B ; C\right]} h\left(X_{n+1} ; \mathfrak{G}\right) .
\end{gathered}
$$

При таком представлении значение $\mathrm{f}_{n}\left[f_{n+1}^{(1)}\right]$ функционала $\mathrm{f}_{n}[\cdot]$ дается суммой

$$
\mathrm{f}_{n}\left[f_{n+1}^{(1)}\right]=\sum_{B \subset I_{n}:|B| \geqslant 1} \sum_{C \subset B} f_{n+1}\left(X_{n+1} ; B ; C\right),
$$

6. Так как каждый класс $\overline{\mathcal{G}}\left[I_{n} ; B ; C\right]$ графов с $n \geqslant 2$, согласно леммам 2.4 и 2.5 , представим в виде дизъюнктивного объединения непустых классов

$$
\overline{\mathcal{G}}\left[I_{n} ; B ; C\right]=\bigcup_{\{B(z) ; z \in C\} \in \mathfrak{D}(B, C)} \overline{\mathcal{G}}\left[I_{n} ; B \mid\{B(z), z \in C\}\right] .
$$

Тогда функции $f_{n+1}\left(X_{n+1} ; B ; C\right)$ представимы в виде следующих сумм:

$$
\begin{gathered}
f_{n+1}\left(X_{n+1} ; B ; C\right)=\sum_{\{B(z) ; z \in C\} \in \mathfrak{D}(B, C)} f_{n+1}\left(X_{n+1} ; B \mid\{B(z), z \in C\}\right), \\
f_{n+1}\left(X_{n+1} ; B \mid\{B(z), z \in C\}\right)=\sum_{\mathfrak{G} \in \overline{\mathcal{G}}\left[I_{n} ; B \mid\{B(z), z \in C\}\right]} h\left(X_{n+1} ; \mathfrak{G}\right) .
\end{gathered}
$$


При этом ввиду линейности функционала $\mathrm{f}_{n}[\cdot]$ имеет место равенство

$$
\mathrm{f}_{n}\left[f_{n+1}\left(X_{n+1} ; B ; C\right)\right]=\sum_{\{B(z) ; z \in C\} \in \mathfrak{D}(B, C)} \mathrm{f}_{n}\left[f_{n+1}\left(X_{n+1} ; B \mid\{B(z), z \in C\}\right)\right] .
$$

7. Наконец, примем во внимание, что каждый класс $\overline{\mathcal{G}}\left[I_{n} ; B \mid\{B(z), z \in C\}\right], n \geqslant 2$, эквивалентен, согласно лемме 2.6, декартову произведению

$$
\overline{\mathcal{G}}\left[I_{n} ; B \mid\{B(z), z \in C\}\right]=\mathcal{F}[B ; n+1] \otimes\left(\bigotimes_{z \in C} \overline{\mathcal{G}}[B(z) ; z]\right),
$$

где $\mathcal{F}_{n}[B ; n+1]$ - класс графов без вершин сочленения над множеством вершин $B \cup\{n+1\}$, $\overline{\mathcal{G}}[B(z) ; z], z \in C$ - набор таких непустых классов, что $\overline{\mathcal{G}}_{z}[B(z) ; z]$ содержит все связные графы с множеством вершин $B(z) \cup\{z\}$ и выделенной вершиной $z \in C$. Тогда

$$
\begin{aligned}
& \mathrm{f}_{n}\left[f_{n+1}\left(X_{n+1} ; B \mid\{B(z), z \in C\}\right)\right]= \\
& \quad=\sum_{\mathfrak{G}_{B} \in \mathcal{F}_{n}[B ; n+1]}\left(\prod_{z \in C} \sum_{\mathfrak{G}(z) \in \overline{\mathcal{G}}_{z}[B(z) ; z]}\right) \mathrm{f}_{n}\left[h\left(X_{n+1} ; \bigvee_{z \in C}\left\{\mathfrak{G}_{B} \vee \mathfrak{G}(z)\right\}\right)\right]
\end{aligned}
$$

Так как (ср. с (5.7))

$$
h\left(X_{n+1} ; \bigvee_{z \in C}\left\{\mathfrak{G}_{B} \vee \mathfrak{G}(z)\right\}\right)=h\left(X(B) ; \mathfrak{G}_{B}\right) \prod_{z \in C} h(X(B(z)) ; \mathfrak{G}(z)),
$$

то, ввиду мультипликативности (5.8), получаем следующее выражение:

$$
\mathrm{f}_{n}\left[h\left(X_{n+1} ; \bigvee_{z \in C}\left\{\mathfrak{G}_{B} \vee \mathfrak{G}(z)\right\}\right)\right]=\mathrm{f}_{|B|}\left[h\left(X(B) ; \mathfrak{G}_{B}\right)\right] \prod_{z \in C} \mathrm{f}_{|B(z)|}[h(X(B(z)) ; \mathfrak{G}(z))]
$$

Тогда на основании (5.12) получаем

$$
\mathrm{f}_{n}\left[f_{n+1}\left(X_{n+1} ; B \mid\{B(z), z \in C\}\right)\right]=\mathrm{f}_{|B|}\left[g_{|B|+1}\right] \cdot \prod_{z \in C} \mathrm{f}_{|B(z)|}\left[\bar{f}_{|B(z)|+1}\right],
$$

где использованы введенные в (5.3) функции $g_{n+1}\left(X_{n+1}\right)$, а также функции

$$
\bar{f}_{B(z) \mid+1}\left(X_{|B|+1}\right)=\sum_{\mathfrak{G}(z) \in \overline{\mathcal{G}}_{z}[B(z) ; z]} h\left(X_{|B|+1} ; \mathfrak{G}(z)\right), \quad z \in C .
$$

В формуле (5.12) в случае, когда $C=\varnothing, \mathfrak{G}_{B}=\mathfrak{G}$, левая часть равенства равна $\mathrm{f}_{n}\left[f_{n+1}\left(X_{n+1}\right)\right]$.

8. На основании формул (5.10), (5.11), (5.13) получаем:

$$
\begin{aligned}
\mathrm{f}_{n}\left[f_{n+1}^{(1)}\left(X_{n+1}\right)\right] & =\sum_{B \subset I_{n}:|B| \geqslant 1} \sum_{C \subset B} f_{n+1}\left(X_{n+1} ; B ; C\right)= \\
= & \sum_{B \subset I_{n}:|B| \geqslant 1} \sum_{C \subset B} \sum_{\{B(z) ; z \in C\} \in \mathcal{D}(B, C)} \mathrm{f}_{n}\left[f_{n+1}\left(X_{n+1} ; B \mid\{B(z), z \in C\}\right)\right]= \\
& =\sum_{B \subset I_{n}:|B| \geqslant 1} \sum_{C \subset B} \sum_{\{B(z) ; z \in C\} \in \mathfrak{D}(B, C)} \mathrm{f}_{|B|}\left[g_{|B|+1}\right] \cdot \prod_{z \in C} \mathrm{f}_{|B(z)|}\left[\bar{f}_{|B(z)|+1}\right]= \\
& =\sum_{m=1}^{n}\left(\begin{array}{c}
n \\
m
\end{array}\right) \sum_{C \subset I_{m}} \sum_{\{B(z) ; z \in C\} \in \mathfrak{D}\left(I_{m}, C\right)} \mathrm{f}_{m}\left[g_{m+1}\right] \cdot \prod_{z \in C} \mathrm{f}_{|B(z)|}\left[\bar{f}_{|B(z)|+1}\right]=
\end{aligned}
$$




$$
\begin{gathered}
=\sum_{m=1}^{n}\left(\begin{array}{c}
n \\
m
\end{array}\right) \sum_{l=1}^{m}\left(\begin{array}{c}
m \\
l
\end{array}\right) \sum_{\left\{B\left(z_{j}\right) ; j \in I_{l}\right\} \in \mathfrak{D}\left(I_{m}, I_{l}\right)} \mathrm{f}_{m}\left[g_{m+1}\right] \cdot \prod_{j=1}^{l} \mathrm{f}_{\left|B\left(z_{j}\right)\right|}\left[\bar{f}_{\left|B\left(z_{j}\right)\right|+1}\right]= \\
=\sum_{m=1}^{n}\left(\begin{array}{c}
n \\
m
\end{array}\right) \mathrm{f}_{m}\left[g_{m+1}\right] \sum_{l=1}^{m}\left(\begin{array}{c}
m \\
l
\end{array}\right) \sum_{\substack{\left\langle k_{1}, \ldots, k_{l}\right\rangle: \\
k_{1}+\cdots+k_{l}=n-m, k_{j} \geqslant 1, j=1, \ldots, l}} \frac{(n-m) !}{k_{1} ! \ldots k_{l} !} \cdot \prod_{j=1}^{l} \mathrm{f}_{k_{j}}\left[\bar{f}_{k_{j}+1}\right] ;
\end{gathered}
$$

здесь $\mathrm{f}_{0}\left[\bar{f}_{|B(z)|+1}\right]=1$ при $B(z)=\varnothing$.

9. Ввиду сходимости степенных рядов, используемых в следующих преобразованиях, согласно (5.9), получаем

$$
\begin{aligned}
\mathrm{S}[z ; \overline{\mathrm{f}}]=\sum_{n=0}^{\infty} \frac{z^{n}}{n !} \mathrm{f}_{n}\left[\bar{f}_{n+1}\right]=1+\sum_{n=1}^{\infty} \frac{z^{n}}{n !} \sum_{s=1}^{n} \frac{1}{s !} \sum_{\substack{l_{j} \geqslant 1, j=1, \ldots, s: \\
l_{1}+\cdots+l_{s}=n}} \frac{n !}{l_{1} ! \ldots l_{s} !} \prod_{j=1}^{s} \mathrm{f}_{l_{j}}\left[\bar{f}_{l_{j}+1}^{(1)}\left(X_{l_{j}}, x_{n+1}\right)\right]= \\
=1+\sum_{s=1}^{\infty} \frac{1}{s !} \sum_{s=n}^{\infty} \sum_{\substack{l_{j} \geqslant 1, j=1, \ldots, s: j=1 \\
l_{1}+\cdots+l_{s}=n}} \prod_{j=1}^{s} \frac{z^{l_{j}}}{l_{j} !} \mathrm{f}_{l_{j}}\left[\bar{f}_{l_{j}+1}^{(1)}\left(X_{l_{j}}, x_{n+1}\right)\right]= \\
=1+\sum_{s=1}^{\infty} \frac{1}{s !} \prod_{j=1}^{s} \sum_{l_{j}=1}^{\infty} \frac{z^{l_{j}}}{l_{j} !} \mathrm{f}_{l_{j}}\left[\bar{f}_{l_{j}+1}^{(1)}\left(X_{l_{j}}, x_{n+1}\right)\right]=\exp \left[\sum_{n=1}^{\infty} \frac{z^{n}}{n !} \mathrm{f}_{n}\left[\bar{f}_{n+1}^{(1)}\left(X_{n+1}\right)\right]\right] .
\end{aligned}
$$

Преобразуем теперь сумму, стоящую в показателе экспоненты, после подстановки в нее значения (5.14) функционала $\mathrm{f}_{n}\left[\bar{f}_{n+1}^{(1)}\left(X_{n+1}\right)\right]$ :

$$
\begin{gathered}
\sum_{n=1}^{\infty} \frac{z^{n}}{n !} \mathrm{f}_{n}\left[\bar{f}_{n+1}^{(1)}\left(X_{n+1}\right)\right]=\sum_{n=1}^{\infty} \frac{z^{n}}{n !} \sum_{m=1}^{n} \frac{n !}{m !} \mathrm{f}_{m}\left[g_{m+1}\right] \sum_{l=1}^{m}\left(\begin{array}{c}
m \\
l
\end{array}\right) \sum_{\substack{\left\langle k_{1}, \ldots, k_{l}\right\rangle: \\
k_{1}+\ldots+k_{l}=n-m, k_{j} \geqslant 1, j=1, \ldots, l}} \prod_{j=1}^{l} \frac{1}{k_{j} !} \mathrm{f}_{k_{j}}\left[\bar{f}_{k_{j}+1}\right]= \\
=\sum_{m=1}^{\infty} \frac{z^{m}}{m !} \mathrm{f}_{m}\left[g_{m+1}\right] \sum_{l=1}^{m}\left(\begin{array}{c}
m \\
l
\end{array}\right) \sum_{n=m}^{\infty} \sum_{\substack{\left\langle k_{1}, \ldots, k_{l}\right\rangle: \\
k_{1}+\cdots+k_{l}=n-m, k_{j} \geqslant 1, j=1, \ldots, l}} \prod_{j=1}^{l} \frac{z^{k_{j}}}{k_{j} !} \mathrm{f}_{k_{j}}\left[\bar{f}_{k_{j}+1}\right]= \\
=\sum_{m=1}^{\infty} \frac{z^{m}}{m !} \mathrm{f}_{m}\left[g_{m+1}\right] \sum_{l=1}^{m}\left(\begin{array}{c}
m \\
l
\end{array}\right) \sum_{\substack{\left\langle k_{1}, \ldots, k_{l}\right\rangle: \\
k_{j} \geqslant 1, j=1, \ldots, l}} \prod_{j=1}^{l} \frac{z^{k_{j}}}{k_{j} !} \mathrm{f}_{k_{j}}\left[\bar{f}_{k_{j}+1}\right]= \\
=\sum_{m=1}^{\infty} \frac{z^{m}}{m !} \mathrm{f}_{m}\left[g_{m+1}\right] \sum_{l=1}^{m}\left(\begin{array}{c}
m \\
l
\end{array}\right) \prod_{j=1}^{l} \sum_{\substack{k_{j}=1 \\
k_{j}}}^{\infty} \frac{z^{k_{j}}}{k_{j} !} \mathrm{f}_{k_{j}}\left[\bar{f}_{k_{j}+1}\right]= \\
=\sum_{m=1}^{\infty} \frac{z^{m}}{m !} \mathrm{f}_{m}\left[g_{m+1}\right]\left(\sum_{k=0}^{\infty} \frac{z^{k}}{k !} \mathrm{f}_{k}\left[\bar{f}_{k+1}\right]\right)^{m}=\sum_{m=1}^{\infty} \frac{z^{m}}{m !} \mathrm{f}_{m}\left[g_{m+1}\right](\mathrm{S}[z ; \bar{f}])^{m} .
\end{gathered}
$$

Тогда

$$
\mathrm{S}[z ; \overline{\mathrm{f}}]=\exp (\mathrm{S}[z \mathrm{~S}[z ; \overline{\mathrm{f}}] ; \mathrm{g}]-1)
$$

Теорема доказана.

6. Заключение. В заключение укажем на некоторые применения описанной в работе алгебраической техники. В равновесной статистической механике классических систем (см. [8]) при 
вычислении уравнения состояния $P(z, T)$ в виде степенного ряда по так называемой активности $z$ возникает величина $\ln \Xi$, где $\Xi$ - статистическая сумма системы, определяемая формулой

$$
\Xi=\sum_{n=0}^{\infty} \frac{z^{n}}{n !} \int_{\Omega^{n}} \exp \left(-\sum_{\{j, k\} \subset I_{n}} U\left(x_{j}-x_{k}\right) / T\right) \prod_{k=1}^{n} d x_{k}=\mathrm{f}[z ; \mathrm{f}],
$$

где $d x$ - мера Лебега в $\mathbb{R}^{3}$, которая инвариантна относительно группы трансляций, $U(x)$ - потенциал взаимодействия в точке $x \in \mathbb{R}^{3}$. Здесь компоненты последовательности $\mathrm{f}$ симметрических функций порождаются функцией $w(x, y)=\exp (-U(x-y) / T)$. Выражение для $P(z, T)$ дается формулой (2.2), в которой коэффициенты так называемого группового разложения определяются функциями последовательности $\overline{\mathrm{f}}$. В терминах введенных величин плотность $\rho$ числа частиц для указанных систем определяется формулой

$$
\rho=\sum_{n=0}^{\infty} \frac{z^{n+1}}{n !} \int_{\Omega^{n}} \bar{f}_{n+1}\left(X_{n+1}\right) \prod_{k=1}^{n} d x_{k}=\mathrm{S}[z ; \overline{\mathrm{f}}] .
$$

Тогда формула (5.4) представляет уравнение $\rho=\exp (\mathrm{S}[z \rho ; \mathrm{g}]-1)$ относительно $\rho$, в котором коэффициенты (называемые неприводимыми интегралами) определяются компонентами последовательности $\mathrm{g}$.

Наконец, укажем простое применение формулы (5.4) в задаче перечисления графов без вершин сочленения (см. [2]).

Теорема 6.1. Пусть в последовательностях $\left\langle N_{n} ; n \in \mathbb{N}\right\rangle u\left\langle M_{n} ; n \in \mathbb{N}\right\rangle$ компоненты $N_{n}-$ числа связных графов с $n \in \mathbb{N}$ вершинами (где $\left.N_{1}=1\right)$ и $M_{m}$ - числа связных графов без вершин сочленения с $m \in \mathbb{N}$ вершинами $\left(M_{1} \equiv 1\right)$. Тогда числа $M_{n+1}$ определяются рекуррентно через $N_{m}, m=1, \ldots, n+1, u M_{m}, m=1, \ldots, n$ по формуле

$$
N_{n+1}=n ! \sum_{s=1}^{n} \frac{1}{s !} \sum_{\substack{\left\langle k_{1}, \ldots, k_{s}\right\rangle, k_{j} \in \mathbb{N}: \\ k_{1}+\cdots+k_{s}=n}} \prod_{j=1}^{s}\left(\sum_{m=1}^{k_{j}} \frac{M_{m+1}}{m !} \sum_{\substack{\left\langle l_{1}, \ldots, l_{m}\right\rangle, l_{i} \in \mathbb{N}_{+}: \\ l_{1}+\cdots+l_{m}=k_{j}-m}} \prod_{i=1}^{m} \frac{N_{l_{i}+1}}{l_{i} !}\right) .
$$

Доказательство. Достаточно положить $\mathrm{f}_{m}\left[g_{m+1}\right]=M_{m+1}$ и $\mathrm{f}_{n}\left[\bar{f}_{n+1}\right]=N_{n+1}$ в формулах $(5.9)$ и (5.14).

Следствие 6.1 (см. [7]). Производящие функции

$$
F(z)=\sum_{n=0}^{\infty} \frac{z^{n}}{n !} N_{n+1}, \quad G(z)=\sum_{n=0}^{\infty} \frac{z^{n}}{n !} M_{n+1}
$$

связаны следующим функииональным уравнением:

$$
F(z)=\exp [G(z F(z))-1]
$$

Доказательство. Утверждение следует из (5.4) при $\mathrm{f}_{m}\left[g_{m+1}\right]=M_{m+1}$ и $\mathrm{f}_{n}\left[\bar{f}_{n+1}\right]=N_{n+1}$.

Замечание 6.1. Ряды (6.2) расходятся в каждой ненулевой точке плоскости $z$, т.е. их нужно рассматривать как асимптотические степенные ряды. Несмотря на расходимость, их допустимо использовать для последовательного вычисления чисел $M_{m}, m \in \mathbb{N}$, на основе производящей функции $F(z)$ чисел $N_{n}$, посредством вычисления производных порядка $n=1, \ldots, m$ (обоснование этой возможности дано в [2]). Например, так как

$$
F(0 ; 1)=1, F^{\prime}(0 ; 1)=1, F^{\prime \prime}(0 ; 1)=N_{3}=4, F^{\prime \prime \prime}(0 ; 1)=N_{4}=38, F^{I V}(0 ; 1)=N_{5}=728,
$$

то применение этой процедуры приводит к следующим значениям:

$$
G(0,1)=1, G^{\prime}(0,1)=M_{2}=1, G^{\prime \prime}(0 ; 1)=M_{3}=1, G^{\prime \prime \prime}(0 ; 1)=M_{4}=10, G^{I V}(0 ; 1)=M_{5}=238 .
$$




\section{СПИСОК ЛИТЕРАТУРЫ}

1. Ван дер Варден Б. Л. Алгебра. - М.: Наука, 1979.

2. Вирченко Ю. П., Остапенко Л. П. Задачи перечисления графов с помеченными вершинами// Науч. вед. БелГУ. Мат. Физ. - 2016. - 44, № 214. - С. 150-180.

3. Гейликман Б. Т. Статистическая теория фазовых превращений. - М.: ГИТТЛ, 1954.

4. Майер Дж., Гепперт-Майер М. Статистическая механика. - М.: Мир, 1980.

5. Малышев В. А., Минлос Р. А. Гиббсовские случайные поля. Метод кластерных разложений. - М.: Наука, 1985.

6. Оре О. Теория графов. - М.: Наука, 1980.

7. Остапенко Л. П., Вирченко Ю. П. Число связных графов без вершин сочленения// Материалы международной конференции «Воронежская зимняя математическая школа С. Г. Крейна-2016». - Воронеж: Научная книга, 2016. - С. 310-314.

8. Рюэль Д. Статистическая механика. Строгие результаты. - М.: Мир, 1971.

9. Харрари Ф. Теория графов. - М.: Мир, 1973.

10. Харрари Ф., Палмер Э. Перечисление графов. - М.: Мир, 1977.

11. Mayer J., Harrison S. F. Statistical mechanics of condensing systems. III// J. Chem. Phys. - 1938. - 6 . - P. 87-100.

12. Mayer J., Harrison S. F. Statistical mechanics of condensing systems. IV// J. Chem. Phys. - 1938. - 6 . - P. 101-104.

Вирченко Юрий Петрович

Белгородский государственный национальный исследовательский университет

E-mail: virch@bsu.edu.ru

Данилова Любовь Петровна

Белгородский государственный национальный исследовательский университет

E-mail: Danilova@bsu.edu.ru 